\title{
A reliability-based approach of fastest routes planning in dynamic traffic network under emergency management situation
}

\author{
Ye Sun* \\ Department of Civil Engineering, Tsinghua University, \\ Beijing 100084, PR China \\ Received 20 August 2011 \\ Accepted 25 November 2011
}

\begin{abstract}
In order to establish an available emergency management system, it is important to conduct effective evacuation with reliable and real time optimal route plans. This paper aims at creating a route finding strategy by considering the time dependent factors as well as uncertainties that may be encountered during the emergency management system. To combine dynamic features with the level of reliability in the process of fastest route planning, the speed distribution of typical intercity roads is studied in depth, and the strategy of modifying real time speed to a more reliable value based on speed distribution is proposed. Two algorithms of route planning have been developed to find three optimal routes with the shortest travel time and the reliability of 0.9 . In order to validate the new strategy, experimental implementation of the route planning method is conducted based on road speed information acquired by field study. The results show that the proposed strategy might provide more reliable routes in dynamic traffic networks by conservatively treating roads with large speed discretion or with relative extreme real speed value
\end{abstract}

Key words:algorithm; reliability of route; modified real time speed; depth first search strategy

\section{Introduction}

Man-made or natural disasters, whether predictable or not, always pose a threat to public safety as well as people's lives and possessions, thus triggering prolonged public concern and heated discussions among researchers and administrators. Many strategies, both empirical and theoretical, have been put forward to mitigate the calamitous consequences that may be caused by the disasters, and researches in the field of transportation play a significant role in exploring effective ways to alleviate the impact of emergencies. Evacuation is a common strategy in emergency management, where the key issue is the proper way to organize different aid agencies and the relocation of the general public. In the realm of transportation, existing evacuation research has been mostly focused on planning stage, such as research on traffic management policies ${ }^{1}$ and origin-destination estimation $^{2-3}$. Additionally, models designed for various emergency cases have been developed to deal with disparate evacuation scenarios, among which the research of Southworth ${ }^{4}$, Urbina and Wolshon ${ }^{5}$, and Alsnih and Stopher ${ }^{6}$ are highly recommended.

Transportation systems are always dynamic, which should not be neglected by scientists and people working in related fields. It is generally acknowledged that even in normal situations, the estimation of traffic flow could hardly be accurate, let alone in accidents and emergencies.

\footnotetext{
*Corresponding author: sunye08@mails.tsinghua.edu.cn
} 
Analyzing and predicting traffic flow based on the concept of dynamic traffic features and uncertain factors are to some extent the most crucial and challenging components in the exploration of emergency management, and failure to do so is always can result in loss of lives as well as public wealth ${ }^{7}$. However, the history of the study of dynamic network is not long. Barrett et $\mathrm{al}^{8}$ reported their study of evacuation management based on real-time dynamic features in 2000, but the solutions and model they proposed are actually based on strategies and techniques which cannot sufficiently satisfy the demand of real dynamic system. Liu et $\mathrm{al}^{7}$ proposed a MRAC framework for real-time traffic management, with a prediction model to generate desired traffic states to achieve optimized system objectives, a real-world traffic model to simulate actual evacuation traffic flows, and an adaptive control model to produce actual traffic control schemes. Liu's work contributed significantly to the improvement of dynamic control of traffic system, but it didn't take sufficient account of the complexity of real traffic network and the uncertainty during the evacuation procedure.

It is also important to note that the development and realization of the proposed strategies and solution frameworks so far are on a large scale decided by the details contained inside the models. It is fundamental that the crucial steps in the whole evacuation procedure should be taken into consideration and can proceed smoothly when designing the models and frameworks. A great deal of innovative research work has been done to achieve this objective. For instance, a network flow model for lane-based evacuation routing was proposed by Cova ${ }^{9}$, which aimed at alleviating traffic delays in evacuation that occurred frequently at intersections. And undoubtedly, searching for the best route is of crucial significance in conducting aid commands efficiently during evacuation procedure, therefore, the development and improvement of optimal route searching algorithm has become a hot research topic in the field of transportation.

Finding the shortest route system is one of the most fundamental and frequently encountered problems in route planning researches, and numerous algorithms are commonly studied and utilized ${ }^{10}$. Dijkstra algorithm is a classical optimal Label-Setting algorithm to solve the one-to-one shortest route problem in a static traffic network $^{11}$. In 1968, Hart proposed the $A^{*}$ algorithm structured on heuristic function which guides the search towards the destination node, thus contributing a great deal to the improvement on searching efficiency ${ }^{12}$. Depth-first search is a general technique used in Artificial Intelligence for solving a variety of problems in planning, decision making, theorem proving, expert systems, etc. And it can examine all the possible routes from an initial node to the destination in a given area with very little memory requirement ${ }^{13}$. Another widely utilized algorithm is ant colony algorithm initially proposed in 1992 by Marco Dorigoin in his $\mathrm{PhD}$ thesis ${ }^{14}$. Inspired by at behavior, the optimization strategy and technique is applied to various field including vehicle routing. ${ }^{15}$ However, basic algorithms and route planning strategies are not sufficient for real problems, to tackle the problem commonly confronted due to the dynamic features of real transportation system, algorithms with the capacity to deal with dynamic network are in great demand. Chabini extended the $A^{*}$ methodology to the shortest route problems in dynamic networks, in which arc travel times are time dependent ${ }^{11}$. And $\mathrm{Fu}$ introduced a new route planning algorithm in dynamic network and restricted areas, and argued that path-planning algorithm should aim at the fastest path, which means the path with the least travel time, in order to take into consideration the actual condition of a real road ${ }^{16}$.

The concept of fastest path in dynamic network is practical, and paves the way for a more direct strategy to analyze optimal path problem ${ }^{17}$. But it is essential to consider uncertainty that exists in the whole process of evacuation process. Even though we can acquire the most current data of traffic flow and the condition of each road we are tracing, we should be aware of the fact that the information we acquire may not be completely reliable, and would change in some way to fit in its own inner regularities. Consequently, we need to examine carefully before the utilization of the currently available figures and resources.

With this understanding, the main objectives of this paper are to develop a new strategy to find three fastest routes from one origin to one destination in a dynamic system, which takes into account the level of reliability of the current data of roads we obtain. The research is set in the background of emergency management procedure, where various aid agencies need the information of reliable fastest route in order to arrive at the incident site as soon as possible. Three paths are searched in this strategy to provide aid agencies with different choices, and enable separate teams from the same origin to perform their tasks 
simultaneously. This paper is structured as follows: the next section presents basic introductions of spot study and speed frequency distributions of typical intercity roads, which is followed by the mathematical model presented to simulate the speed distribution. In section 3, approaching of optimal routes planning is discussed, and two algorithms in the strategy are discussed in detail. After that experimental implementations of the new strategy is conducted based on simulated map of the district selected and statistical data acquired by field study. Finally, some concluding remarks and future work are summarized in brief.

\section{Spot speed studies and frequency distribution curve}

\subsection{Using the normal distribution in analysis of spot speed data}

Spot speed has been previously defined as the average speed of vehicles passing a point on a highway. When the speeds of individual vehicles are measured at a given spot or location, the result is a distribution of speeds, as no two vehicles will be travelling at exactly the same speed. Therefore, one of the basic objectives of spot speed study is to clearly describe the distribution of speed, and to determine an adequate mathematical model to analyze the observed distribution.

In a field study, large amounts of samples are collected, and the number of individual vehicles travelling at particular ranges of speed is recorded. Speed data is generally presented as the frequency of occurrence within predetermined ranges of speed, and mathematical descriptions of the distribution are proposed. Most speed distributions are statistically normal (i.e., they can be reasonably represented by a normal distribution), and some other distributions can be described using models modified on the basis of a normal distribution ${ }^{18-19}$.

In order to use a normal distribution to represent the observed distribution of speed data, some key statistics should be determined, among which the most decisive are the mean and the standard deviation of the distributed speed values, represented by $\mu$ and $\sigma$ respectively. In practical terms, the true value, $\mu$ and $\sigma$, are unknown. What results from a spot speed study are estimates of true mean and standard deviation of the distribution based on a measured sample, $s$ and $\bar{x}$ :

$$
\bar{x}=\frac{\sum_{i} n_{i} S_{i}}{N}, s=\sqrt{\frac{\sum_{i} n_{i}\left(S_{i}-\bar{x}\right)^{2}}{N-1}}
$$

where $n_{i}$ is the frequency of observations in speed group I, $S_{i}$ is the middle speed of speed group $i$, and $\mathrm{N}$ is the total sample size.

It is vital to note that the sample size is of significance importance to the precision of the estimation. Given that the precision or tolerance $(e)$ of the estimate is the \pm range around the real mean, and a confidence level of $99.7 \%$ is required (i.e. the possibility of $\bar{x}$ in the range of $\mu \pm e$ is $99.7 \%$ ), the required sample size $\mathrm{N}$ can be calculated by equation below:

$$
e=3.00 \frac{s}{\sqrt{N}}
$$

Most observed speed distributions have standard deviations that are close to 5 , as this represents most driver behavior patterns reasonably well. As a consequence, most field studies yield similar standard deviations (Roger, 2004).

\subsection{Conservative planning with 10th percentile speed}

Once the mathematical models of spot speed data acquired in a normal condition are established, they can be stored by traffic management authorities to describe common properties of the paths where observation spots are set. Speed distribution model of a particular spot is representative of speed distribution of the path where the spot lies in an intercity network, since intersections are relatively close to each other, and speeds of vehicles on most spots along one path are approximately similar during a small period of time. Admittedly, at intersections speed will fluctuate due to traffic control devices such as traffic lights, but it can be mitigated in a huge traffic network, where influences of various intersections could be neutralized by each other.

Although estimated mean value of speed distribution is universally used in assessments of road conditions, it is unsafe to draw the conclusion that the speed of vehicles on the road will stably stay in a range near the estimated meal value. In order to conservatively estimate the condition of a road, a 10th percentile speed is proposed. On a particular road, only 10 percent of vehicles run at a speed smaller than 10th percentile speed of the road (Fig. 1.). 


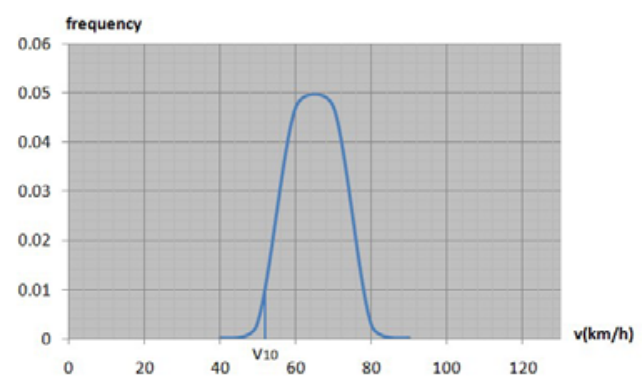

Fig. 1. 10th percentile speed in a normal speed frequency distribution.

Suppose the frequency distribution function of the speed of a road during a particular time period is $f(x), 10$ th percentile speed $\left(v_{10}\right)$ is acquired by integration below:

$$
\int_{-\infty}^{v_{10}} f(x) d x=0.1
$$

For a road with a given length $(d)$ in a traffic network, when $v_{10}$ is calculated, the 90th percentile travel time $\left(t_{90}\right)$ can be determined as follow:

$$
t_{90}=\frac{d}{v_{10}}
$$

which means that 90 percent of the vehicles can pass the road within the $90^{\text {th }}$ percentile travel time.

The introduction of $v_{10}$ and $t_{90}$ enables us to make route planning in a more conservative way, and optimal route searching algorithm will be more conscious when dealing with paths whose speed distribution are relatively disparate. To distinguish algorithms that use $v_{10}$ and $t_{90}$ in their computing procedure and tradition algorithms, we name the latter 'algorithms with the reliability of 90 percent', and routes found based on them 'routes with the reliability of 90 percent'. It is necessary to stress that 90 percent is not a fixed value in the concept of conservative planning, and we can adjust the percentage based on the level of confidence we demand. The more reliability we want, the smaller percentage speed value we assign and utilized in the route planning algorithms.

\section{Strategy of reliable optimal route planning in a dynamic network}

\subsection{Fastest path finding algorithm with reliability of 90 percent}

This algorithm-we can name it $G$ algorithm for convenience-aims at finding 10 routes with the least length of travel time and reliability of 90 percent in a traffic network based on the average velocity of each road in the network in particular time periods, which is static data resource stored in the database of traffic management authorities. When emergencies are reported, emergency management system could instantly present 10 routes with reliability of ninety percent, which could be conducted in a rapid speed and be realized before the expansion of negative influences caused by emergencies. G algorithm is a modified DFS with concepts of time cost generated from $\mathrm{A}^{*}$ algorithm, which is presented in detail as bellow. In $A^{*}$ algorithm, a cost function is defined as ${ }^{12}$ :

$$
\hat{F}_{q}=\hat{T}_{q}+\hat{e}_{q E}
$$

where $q$ is the current expending node (current Node), $\widehat{F}_{q}$ is the estimation of the minimum travel cost among all the paths from origin node $O$ to destination node $D$ that go through $q, \widehat{T}_{q}$ is the upper bound on the minimum travel cost from origin node $O$ to node $q, \hat{e}_{q E}$ is the estimation of the minimum travel cost from node $q$ to destination node $D$.

In order to find out routes with the least travel time, we modify the cost function, which could be presented as follow, measured by the length of time:

$$
\hat{F}_{q}=\hat{T}_{q}+\hat{e}_{q E}
$$

In this function, $\hat{F}_{q}$ is the estimation of the minimum travel time among all the paths from origin node $O$ to destination node $D$ that go through $q, \widehat{T}_{q}$ is the travel time (timeCost) with reliability of $90 \%$ of current route generated from origin node $O$ to node $q$ (currentPath), which is defined as

$$
\widehat{T}_{q}=\sum_{i=0}^{q-1} \frac{d_{i, i+1}}{v_{10, i}}
$$

where $d_{i, i+1}$ is the Euclidean distance between node $i$ and node $i+1$ on the route connecting origin point $\mathrm{O}$ and node $\mathrm{q}$, and $v_{10, i}$ is the average velocity on the road whose endpoints are node I and node $i+1$ respectively.

$\hat{e}_{q E}$ is defined as the minimum possible travel time (minTimeCost), which is determined as

$$
\hat{e}_{q E}=\frac{d_{j E}}{v_{10, \max }}
$$

where $d_{j E}$ is the Euclidean distance between node $j$ and node $D$, and $v_{10, \max }$ is the maximum value of average velocity with reliability of $90 \%$ among all the roads in the traffic network. 
In the depth-first search in $G$ algorithm, the search begins by expanding the initial node (origin node $O$ ), and at each later step, one of the previously generated node is expanded until the destination node $D$ is found, and nodes generated by the expansion of currentNode is called nextNode. In the searching procedure, DFS use $\hat{F}_{q}$ to assess the possibility that a particular node will be on the optimal route by comparing the value of $\hat{F}_{q}$ to the calculated time cost ( $T_{D i}$ for route $\left.i\right)$ of the found routes stored in a set named PATH, which is on the basis of the concept of heuristic search. The process $\mathrm{G}$ algorithm takes to find the fastest path could be described as pseudo-code below:

Initialization: visited[originNode] $=$ true; currentPath $[0]=$ originNode; searchedDepth $=0$;

void DFS(int currentNode) \{

if ( timeCost(currentPath) $\quad+$ minTimeCost(currentNode, destinationNode) $>\max \{$ timeCost(PATH) $\})\{$

return;

if $($ currentNode $==$ destinationNode $)\{$

if ( number of paths in PATH $<10$ or timeCost(currentPath) $<\max \{$ timeCost(paths in PATH) $\}$ ) \{

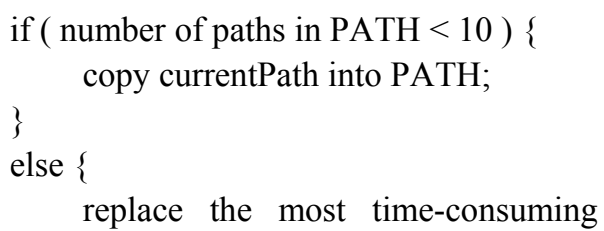

replace the most time-consuming path in PATH with currentPath;

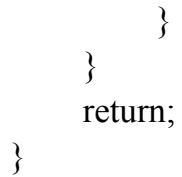

for ( every sibling 'nextNode' of currentNode ) \{ if (nextNode is farther from destinationNode than currentNode and so does the last expansion) \{

// We are very probably in a wrong direction, so drop it

drop this 'nextNode', and turn to the next sibling of currentNode;

\}

else \{
searchedDepth++; currentPath [searchedDeapth] = nextNode; visited[nextNode] = true;

DFS( nextNode ); visited $[$ nextNode $]=$ false; searchedDepth--;

$$
\text { \} }
$$

$\mathrm{G}$ algorithm is a heuristic algorithm that instructs the system to search the optimal routes in a more efficient way. Since it is required that 10 paths be selected out, the searching procedure becomes more complex than traditional optimal route finding algorithms. To decrease time consumed in computing process, a set of routes named PATH is proposed to store the found routes with relatively small travel time (time cost), which enable the system to give up a particular node $\mathrm{j}$ reached from the expansion when $\hat{F}_{j} \geq \max \left\{T_{D i}, \forall\right.$ path $\left.h_{i} \in P A T H\right\}$, thus enhancing the speed when searching for solutions. Meanwhile, it is also of significant importance to point out that the reliability all the paths founded by $\mathrm{G}$ algorithm can be guaranteed. We use $v_{10}$ to calculate the length of time, which ensures that for each small block between the intersections, the possibility of real travel time longer than calculated value in $\mathrm{G}$ algorithm is only $10 \%$.

\subsection{Selection of the fastest route based on the modification of current speed value}

When 10 routes are found by $\mathrm{G}$ algorithm based on the stored data and mathematical model, the paths that aid agencies will probably go along are simultaneously determined. Real time average speed data of these potential paths is acquired by devices that trace the dynamic status of traffic flow, and three optimal routes are then found on the basis of the real time data, and the strategy used to complete this task is named $\mathrm{F}$ algorithm in this paper. This step uses real time average speed to determine the optimal routes, which enables the emergency management system to integrate time dependent factors into the properties of the roads which are acquired using statistic strategies in a dynamic traffic network.

It is generally acknowledged that even though we have access to the data of real time average speed, we cannot precisely predict how the figures will change by the flow of time, and it is unlikely that we update data every single 


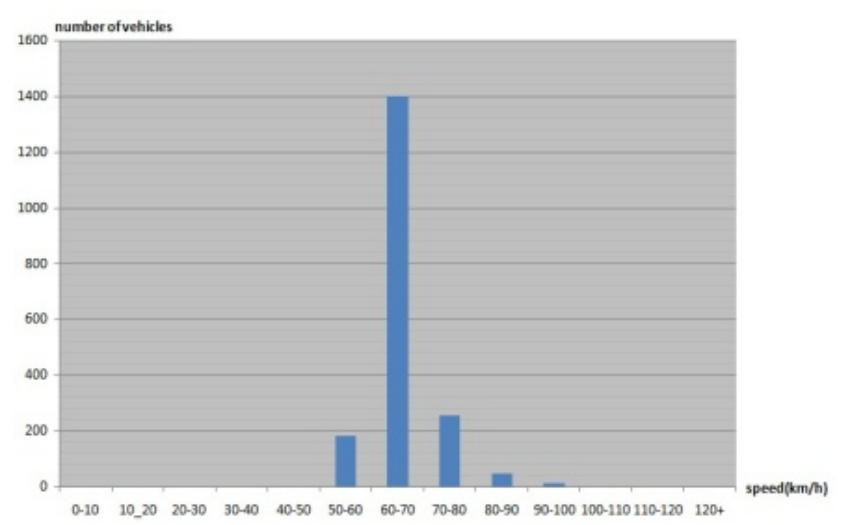

Fig. 2. Speed distribution of a typical road in Beijing from 12PM to $2 \mathrm{AM}$.

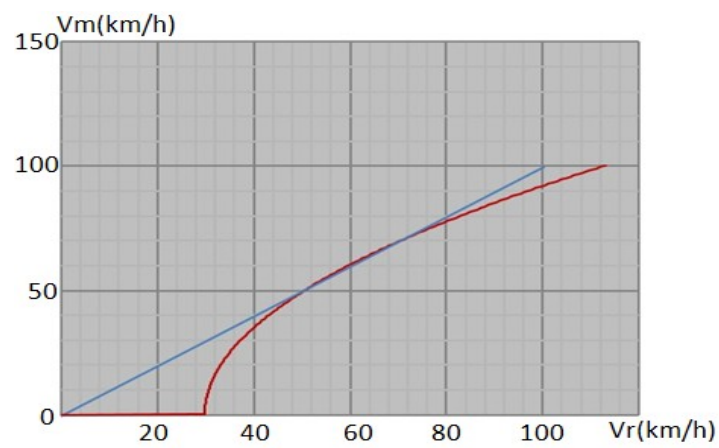

Fig. 3. Modification function based on speed distribution in Fig.2

minute during the emergency management procedure. Consequently, when a real time average speed value is acquired, we should again consider its reliability based on the frequency distribution function and modify the value before applying it to route planning algorithm.

In this paper, we discuss algorithms that modify real time speed values of roads with normal speed distribution and double-peak speed frequency distribution which can be simulated by the superimposition of two normal distribution functions.

Fig. 2. is the bar chart of the speed distribution of a typical road in Beijing from 12PM to 2AM in October, $2007^{20}$, which could be described using a normal distribution model, and the strategy is presented in 2.1. When a real time speed value is acquired, judgment of its reliability should first be conducted. If the value of current average speed is approximately close to the estimated mean speed of the road, it can be inferred that the possibility of the vehicles on this road to reach this speed value is relatively large, thus no big modification is demanded. However, considering the situation when the value of real time average speed is bigger than the estimated mean speed value, the possibility that the speed will drop to a smaller value cannot be ruled out, and it will be more conservative to modify the acquired value downward. Similarly, if we find the real time value smaller than mean value, it is reasonable that we modify it to a bigger value. However, it should also be considered that if the value of real time average speed is so small that it exceeds a tolerance range, it is of all likelihood that the road is suffering from heavy traffic congestion. As a result, we should rule out this road when determining the optimal routes.

The foregoing concept can be realized by a modification function that defines the relationship between real time average speed $\left(v_{r}\right)$ and the modified value $\left(v_{m}\right)$. In order to satisfy the objectives of modification presented above, let

1) $v_{m}=f\left(v_{r}\right)=v_{r}$, when $v_{r}=E$, where $E$ is the mean speed value.

Let $v_{m}<v_{r}$, when $v_{r}>E$, and $v_{m}>v_{r}$, when $v_{r}<E$

2) $v_{m}=f\left(v_{r}\right)=v_{r}$, when $v_{r}=E-1.645 \sigma$

Let $v_{m}>v_{r}$, when $v_{r}>E-1.645 \sigma$.

And when $v_{r}<E-1.645 \sigma$ (the probability is only 5\%), let $v_{m}<<v_{r}$, which makes the road unlikely to be selected. A quadratic function is introduced to describe the relationship between $v_{r}$ and $v_{m}$ :

$$
v_{r}=A v_{m}^{2}+B v_{m}+C
$$

To simplify the function, let $B=0, A$ and $C$ can be determined by 1) and 2) stated above. Given the normal distribution model determined by figures in Fig.2., the function curve of $f\left(v_{r}\right)$ is presented in Fig.3., where $A=8.32 \times 10-3, C=29.83$.

Fig.4. is the bar chart of the speed distribution of a typical road in Beijing from 6AM to 8AM in October, 2007 (Li, 2007), which can be simulated as two normal distribution models. And the frequency distribution function is presented as below:

$$
f(x)=\lambda \frac{1}{2 \pi \sigma_{1}} e^{\frac{\left(x-E_{1}\right)^{2}}{-2 \sigma_{1}^{2}}}+(1-\lambda) \frac{1}{2 \pi \sigma_{2}} e^{\frac{\left(x-E_{2}\right)^{2}}{-2 \sigma_{2}^{2}}}, 0<\lambda<1
$$

where $E_{1}, E_{2}$ are the mean values of the two normal distribution models, and $\sigma_{1}, \sigma_{2}$ are the standard deviations. It is essential to judge if the two normal distribution models are dependent before defining the modification function. 


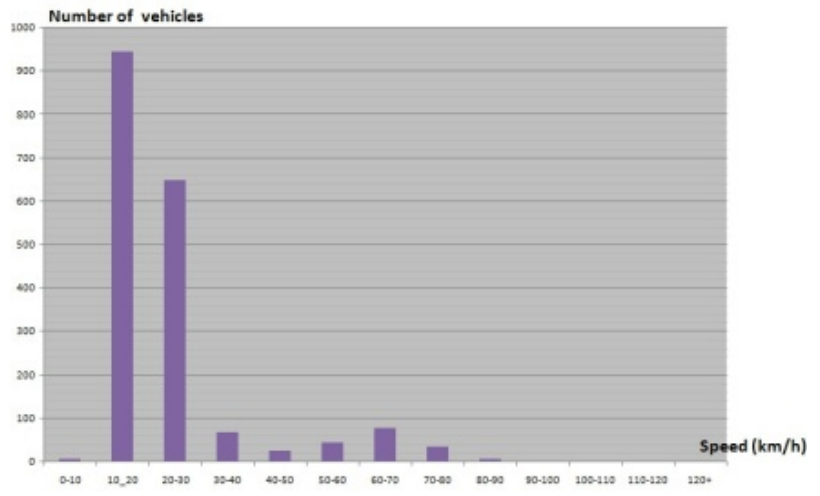

Fig. 4. Speed distribution of a typical road in Beijing from $6 \mathrm{AM}$ to $8 \mathrm{AM}$

1) If $E_{1}+\beta \sigma_{1} \leq E_{2}-\beta \sigma_{2}$

Let $\beta=0.84$, which ensures that the two normal distribution models can be treated as independent. In order to be more conservative, we determine $v_{m}$ by the equation

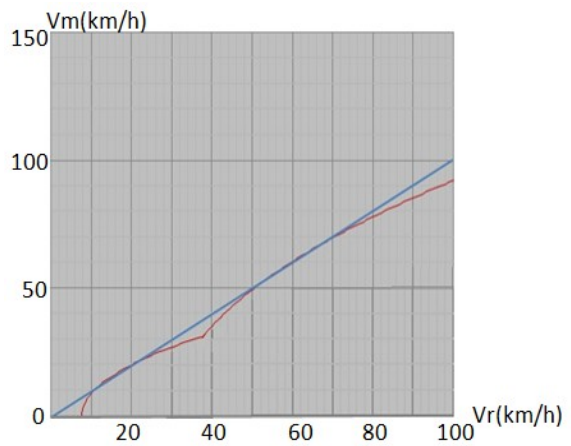

below:

$$
v_{r}=\min \left\{A_{1} v_{m}^{2}+C_{1}, A_{2} v_{m}^{2}+C_{2}\right\}
$$

where $A_{1}, A_{2}, C_{1}, C_{2}$ are the coefficients determined by the two dependent normal distributions models.

2) If $E_{1}+\beta \sigma_{1}>E_{2}-\beta \sigma_{2}$

In this situation, the two normal distribution models are mixed on a relatively large scale, thus cannot be simulated as two independent peaks. We conservatively select the smaller value of determined by two normal distribution models, and if we choose the smaller value when modifying speed value of all the observed roads in the network, it is evident that the error can be mitigated. Thus, $v_{m}$ is calculated by the equation below:

$$
v_{r}=\max \left\{A_{1} v_{m}^{2}+C_{1}, A_{2} v_{m}^{2}+C_{2}\right\}
$$

where $A_{1}, A_{2}, C_{1}, C_{2}$ are the coefficients determined by the two dependent normal distributions models.

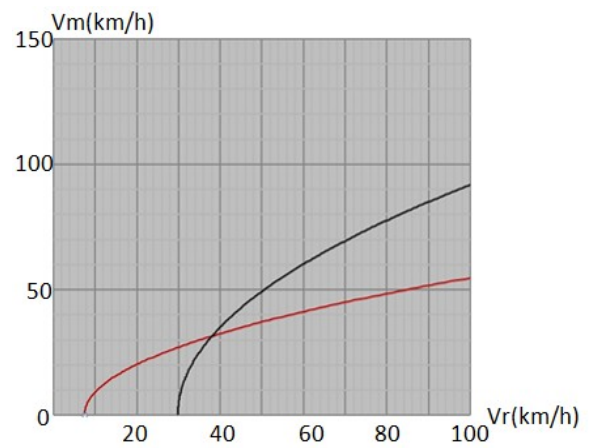

Fig. 5. Modification functions based on speed distribution in Fig.4 before and after superimposition.

Fig.5. (left) is the function curves of modified speed based on the data provided in Fig.4. before superimposition, and Fig.5. (right) shows the superimposed curves. In this model, $A_{1}=8.32 \times 10^{-3}, C_{1}=29.83, A_{2}=0.031, C_{2}=7.601$. Function curves of $v_{m}=v_{r}$ is also illustrated as a reference of the situation before modification.

When modified speeds of all the potential roads are selected, estimated time cost $\left(t_{e}\right)$ of each route is calculated as bellow:

$$
t_{e}=\sum_{i} \frac{d_{i}}{v_{m, i}}
$$

where $d_{i}$ is the distance road $i$ in the found route provided in $\mathrm{G}$ algorithm, which can be regarded as the Euclidean distance between two nearby intersections in a traffic network, and $v_{m, i}$ is the modified speed of road $i$.
Based on $t_{e}$, emergency management systems can select 3 routes with the shortest travel time among the 10 routes acquired in the previous step, and immediately provide aid agencies with the route information.

\section{An experimental study of computer implication based on a real traffic network}

\subsection{District selected in the experimental study and introduction to the implementation system.}

$\mathrm{G}$ algorithm and $\mathrm{F}$ algorithm discussed in the previous section have been implemented for the purpose of computational test, and traditional DFS strategy using estimated mean speed value is applied as a comparison to the proposed algorithms. To conduct the experimental study, an intercity traffic network in Beijing is selected 
and the map is simulated in AutoCAD. The selected area is the neighborhood of the Workers Stadium, which is to the north of Chang'an Avenue, between the east second and third ring roads, with the total area of $16.92 \mathrm{~km}^{2}$. The area selected is typical in downtown Beijing, which ensures that the transport characteristics of roads in this area are among the average level, and consequently could be estimated based on the representative data of road characteristics in Beijing. Meanwhile, since many public activities are held in the Workers Stadium, study of route planning for emergency management is more useful and practical considering the fact that there is a higher possibility that emergencies will happen in the area near the Workers Stadium.

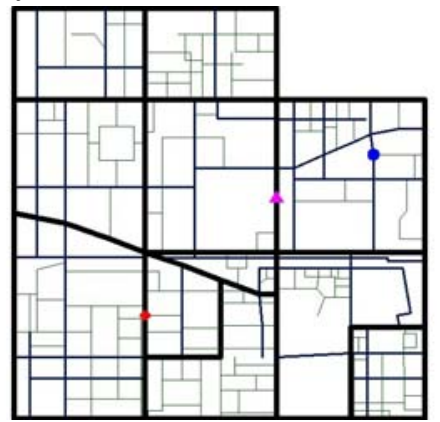

Fig. 6. Simulated map of selected district in experimental implementation.

The simulated map is illustrated in Fig.6. Lines with the largest width stand for expressways whose average speed is usually the fastest among other kinds of roads on this map, and lines with medium width represent trunk roads. Correspondingly, lines with the smallest width stand for sub-trunk roads with relatively lowest average speed among roads showed on the map. As for aid agencies, three most typical agencies are selected to simulate the real situation. The circle, triangle and rhombus stand for fire agency, police station and hospital respectively, which are marked on the map. In the implementation system, emergency spot is generated randomly, and is marked by a star shape sign.
The speed information of expressways, trunk roads and sub-trunk roads are acquired by a field study conducted in October, 2007 by a group of transportation research agency in Tsinghua University (Li, 2007). The field study investigated speed of all vehicles that passed through Yuetan North Bridge and Fuchengmen Bridge, and simulated the distributions of vehicle speed on expressways, trunk roads and sub trunk roads during the twelve periods of a day, which is presented in Table 1. The results are typical for districts between second and third ring roads in the north part of Beijing.

Table 1. Distributions of vehicle speed on expressways, trunk roads and sub trunk roads

\begin{tabular}{c|cccccccccc}
\hline \multirow{2}{*}{ Time period } & \multicolumn{4}{c}{ Expressway } & \multicolumn{3}{c}{ Trunk road } & \multicolumn{3}{c}{ Sub-trunk road } \\
\cline { 2 - 10 } & E1 & $\sigma 1$ & $\lambda 1$ & E2 & $\sigma 2$ & $\lambda 2$ & E1 & $\sigma 1$ & E1 & $\sigma 1$ \\
\hline 12PM-2AM & 65 & 6 & & & & & 45 & 6 & 35 & 6 \\
2AM-4AM & 70 & 3 & & & & & 50 & 3 & 40 & 3 \\
4AM-6AM & 55 & 5.5 & & & & & 45 & 5.5 & 30 & 5.5 \\
6AM-8AM & 30 & 6 & 0.4 & 60 & 8 & 0.6 & 30 & 6 & 20 & 6 \\
8AM-10AM & 30 & 7 & 0.7 & 65 & 7 & 0.3 & 30 & 7 & 20 & 7 \\
10AM-12AM & 25 & 7 & 0.7 & 60 & 7 & 0.3 & 25 & 7 & 25 & 7 \\
12AM-2PM & 30 & 7 & 0.6 & 70 & 8 & 0.4 & 30 & 7 & 20 & 7 \\
2PM-4PM & 20 & 6 & 0.8 & 65 & 6 & 0.2 & 20 & 6 & 20 & 6 \\
4PM-6PM & 20 & 8 & 0.8 & 60 & 7 & 0.2 & 20 & 5 & 20 & 5 \\
6PM-8PM & 20 & 8 & 0.4 & 70 & 8 & 0.6 & 20 & 8 & 20 & 8 \\
8PM-10PM & 30 & 6 & 0.3 & 70 & 8 & 0.7 & 30 & 6 & 30 & 6 \\
10PM-12PM & 80 & 9 & & & & & 60 & 9 & 50 & 9 \\
\hline
\end{tabular}


The implementation system is written in $\mathrm{C}++$ based on MFC (Microsoft Foundation Classes), which is structured by three layers: data layer, logic layer and display layer. The data layer reads information from the map, and transforms it into data that can be recognized by the system. 10th percentile speed $\left(v_{10}\right)$ and real speed $\left(v_{r}\right)$ information of different roads is written in speed.txt and realspeed.txt respectively, which are read by data layer during the path planning procedure. The logic layer realizes the processes of $\mathrm{G}$ algorithm and $\mathrm{F}$ algorithm. Finally, optimal routes are presented on the map by the display layer. Meanwhile, when the routes are found (both the ten routes acquired by $\mathrm{G}$ algorithm and the three routes selected by $\mathrm{F}$ algorithm), the system will automatically generate a text file reporting the nodes along the routes as well as the travel time of each route.

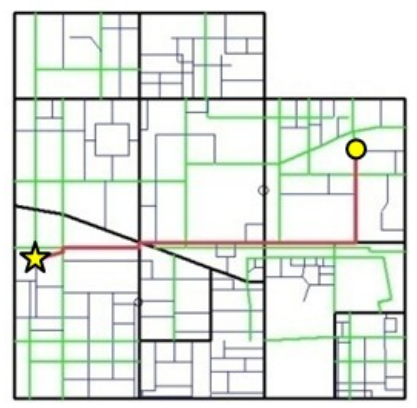

Fig. 6. Route found by traditional DFS in experiment 1 .

\subsection{Experiments of the optimal routes finding}

\subsubsection{Experiments of the routes finding in a normal and stable traffic condition.}

This experiment is set under a condition when all the roads in the map have the real time average speed within the range between 40th and 60th percentile speed. Suppose the time period is $12 \mathrm{PM}-2 \mathrm{AM}$, and the aid agency selected is the fire agency. The real speeds of three different kinds of roads randomly selected from $v_{40}$ and $v_{60}$ are written in realspeed.txt as shown in Table 1.1. It is evident that in real situation, it is unlikely that the real speeds of all the roads within the same category are exactly the same. We usually acquire speed information of each road, and write the information into the emergency management system for route planning. However, to ensure the running speed of this experimental system, we simplify the problem by assigning the same real speed value to the same kind of roads.

Table 1.1. Speed information in experiment 1

\begin{tabular}{cccc}
\hline 12PM-2AM & Expressway & Trunk road & Sub-trunk road \\
\hline$v_{50}$ & 65 & 45 & 35 \\
$v_{10}$ & 52.284 & 37.284 & 27.284 \\
$v_{r}$ & 64.75 & 45.12 & 34.94 \\
$v_{m}$ & 64.77 & 45.11 & 34.95 \\
\hline
\end{tabular}

As shown in Table 1.1, the level of discretion is the same for the three different kinds of road ( $\sigma$ equals). Consequently, when considering the 10th percentile speed, the disparity of the three speed values corresponds with the mean speed. Since the traffic situation is relatively stable, $v_{r}$ is close to the mean value. As a result, the modified speed values approximately equal to the acquired real speed. In this situation, the found optimal routes (Fig.7.) are almost the same as those found by traditional DFS using $v_{50}$ (Fig.6.). The nodes that the three found route go through are listed in Table 1.2, which also presents the Path Cost. In this and the following two examples, Route1, Route 2 and Route 3 are listed by the order of their Time Cost, from the smallest to the largest, and Route 0 is the route found by traditional DFS.
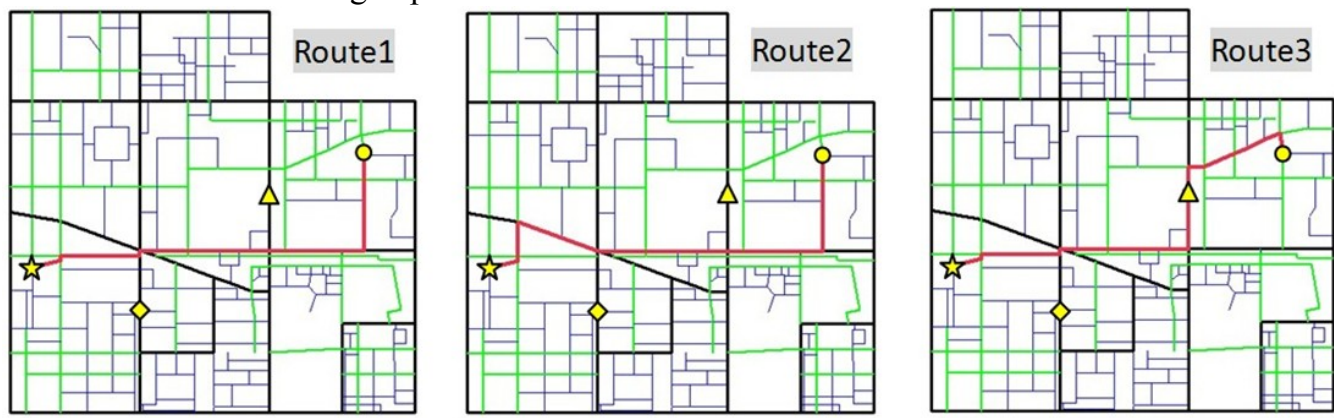

Fig.7. Route found by $\mathrm{G}$ algorithm and $\mathrm{F}$ algorithm in experiment 1 
Table1.2. Nodes that the three found route go through in experiment 1

\begin{tabular}{lrc}
\hline & Nodes & PathCost \\
\hline Route1 & 3722222235420333231302928272624304314367 & 74.5559 \\
Route2 & 372222223222120333231302928272624304314367 & 76.5221 \\
Route3 & 37222222354203332313029282110732 & 77.5507 \\
Route0 & 3722222235420333231302928272624304314367 & 74.5171 \\
\hline
\end{tabular}

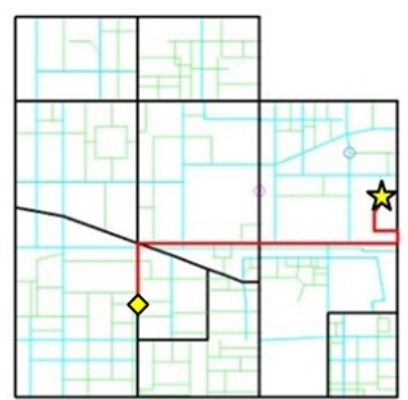

Fig. 8. Route found by traditional DFS in experiment 2 .

\subsubsection{Experiments of the routes finding considering roads with discrete speed distribution.}

This experiment is set under a situation when one particular type of road has discrete speed discretion. Consider the traffic network during peak hours (4PM-6PM) in the afternoon, when three types of roads are in a relatively ultra saturated condition. An emergency happened on the block in the south east part of the selected area, and ambulances are in urgent demand, which calls for immediate route planning from emergency spot to the hospital. At this time, situation on expressways is a complexity, where vehicles' speed fluctuates in a considerable range. Speed information in this example is shown in Table 2.1.

Table 2.1. Speed information in experiment 2

\begin{tabular}{cccc}
\hline 12P M-2AM & Expressway & Trunk road & Sub-trunk road \\
\hline$V_{50}$ & 21.91 & 20 & 20 \\
$V_{10}$ & 10.80 & 13.58 & 13.58 \\
$V_{r}$ & 23.00 & 21.12 & 22.87 \\
$v_{m}$ & 21.92 & 22.03 & 22.16 \\
\hline
\end{tabular}

As shown in Table 2.1, from $4 \mathrm{pm}$ to $6 \mathrm{pm}$, the frequency distribution of average speed on expressways is discrete, which can be simulated by the model of two independent normal distributions. We can infer from the value of 10th percentile speed that conservatively thinking, expressways are not the priority for optimal path planning since their average speed may easily drop to the lowest value. The experiment results are shown in Fig.9. and Table 2.2, as compared to route found by traditional DFS (Fig.8.), it is illustrated that expressways are on a large scale avoided. It may sound beyond common sense when the results are acquired, but it reasonably accounts for uncertainties in the dynamic traffic network, and presents more reliable routes for the aid agencies.

Table2.2. Nodes that the three found route go through in experiment 2

\begin{tabular}{cccc}
\hline & Nodes & PathCost \\
\hline Route1 & 362363365366402539353354296295299298301300357 & 174.156 \\
Route2 & 1051042603403391424724533833724925950 & \\
Route3 & 3623633653664025393533542961061051042603403391424724533833724925950 & 174.176 \\
Route0 & 3623633653664025393533542961061051042603403391424724533833724925950 & 174.182 \\
\hline
\end{tabular}
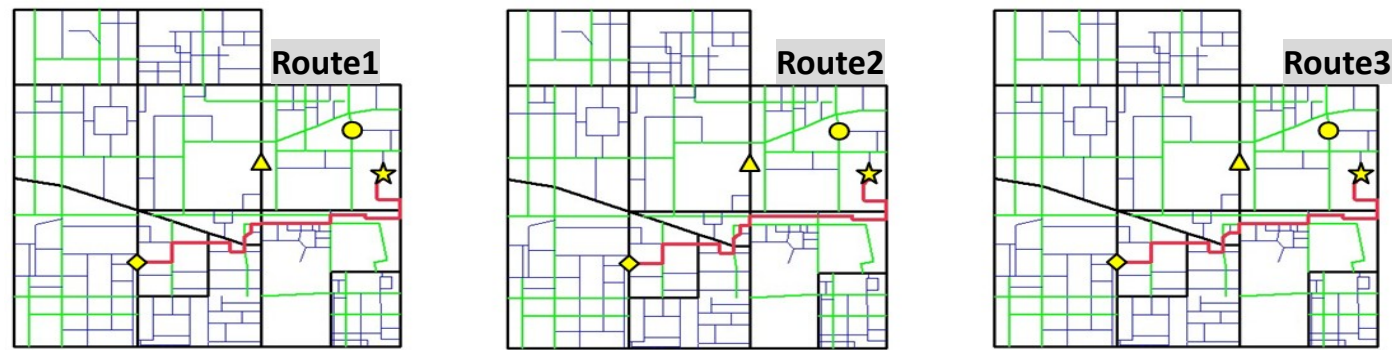

Fig.9. Route found by $\mathrm{G}$ algorithm and $\mathrm{F}$ algorithm in experiment 2 


\subsubsection{Experiments of the routes finding with acquired real speed of extreme value.}

This experiment is set under a situation when the real speed value acquired of one particular type of road is extremely large or small. Based on the frequency distribution function of a road, we can easily find out the proportion of situations when the average

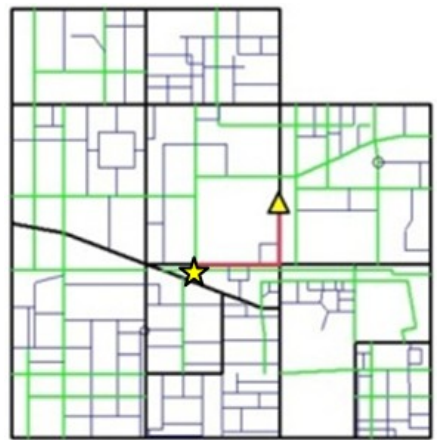

Fig. 10. Route found by traditional DFS in experiment 3 .

vehicle speed on this road is smaller or larger than the real time value, thus determining if the lately obtained value is sufficiently reliable. If a real time speed data acquired is too optimistic or pessimistic, it is highly recommended that we modify the value to a more eclectic value, which makes the obtained value safer to utilize. Consider the traffic network during early hours of the day (4AM-6AM) in the morning, when an accident happened and the police station received the command to reach the accident spot as soon as possible. When obtained real time traffic information shows that the vehicles on a particular trunk road are currently running at an extremely high speed, we should not neglect the fact that this situation may not remain stable in a dynamic network.

The speed information is shown in Table 3.1, and results of generated routes are presented in Table 3.2 and Fig.11. Table 3.1. Speed information in experiment 3

\begin{tabular}{cccc}
\hline 12PM-2AM & Expressway & Trunk road & Sub-trunk road \\
\hline$v_{50}$ & 55 & 45 & 30 \\
$v_{10}$ & 47.93 & 37.93 & 22.93 \\
$v_{r}$ & 55.20 & 55.32 & 34.74 \\
$v_{m}$ & 55.18 & 53.50 & 33.78 \\
\hline
\end{tabular}

It is shown in the results that not all the paths selected from east to west are trunk roads whose real speed value is extremely high (approximately 97th percentile speed). Besides route found by traditional DFS (Figure 10), the found optimal route of the new strategy with the smallest Time Cost is formed still mainly by expressways, whose high real speed value is more reliable when considering the dynamic feature of the traffic network. When referring to the modified speed, it is important to see that the modified speed value of trunk roads is smaller $(53.50 \mathrm{~km} / \mathrm{h})$ than that of expressways $(55.18 \mathrm{~km} / \mathrm{h})$, although the reverse is true for real speed value.

Table3.2. Nodes that the three found route go through in experiment 3

\begin{tabular}{ccc}
\hline & Nodes & PathCost \\
\hline Route1 & 262323130292821 & 22.88 \\
Route2 & 2622642631062821 & 23.26 \\
Route3 & 2622643130292821 & 23.59 \\
Route0 & 262323130292821 & 23.13 \\
\hline
\end{tabular}
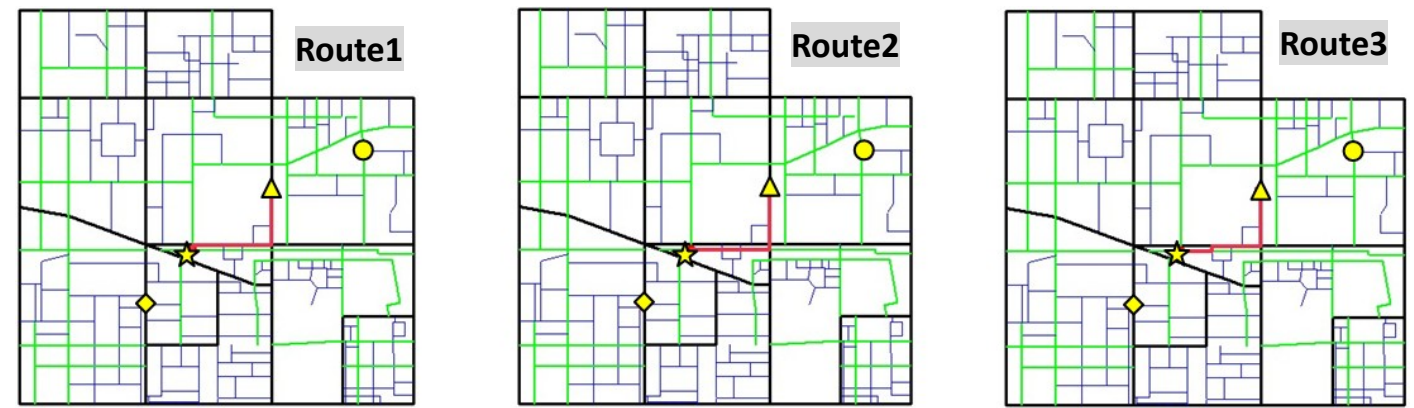

Fig.11. Route found by $\mathrm{G}$ algorithm and $\mathrm{F}$ algorithm in experiment 3 


\section{Concluding remarks and future work}

Dynamic features of traffic network have long been the focus of research concern, and uncertain factors are essential issues that cannot be neglected in route planning strategies, especially when an emergency happens. This paper has proposed a strategy that simultaneously takes into account the dynamic characteristics and the level reliability for optimal route finding against the background of emergency management. Definitions or routes with reliability of 90 percent as well as a recommended mathematical model simulating speed distribution are first presented. In this process, G algorithm is introduced to determine 10 optimal routes with reliability of 90 percent, and $\mathrm{F}$ algorithm is utilized to select 3 routes from the 10 found routes based on the obtained real time speed values. The reliability of speed value and the modification of real time value are determined fundamentally by the speed frequency distribution models of various roads, which are established on the basis of the statistical data obtained by field studies. Meanwhile, experimental implementation of the route planning strategy is conducted, with regarding to three disparate set situations. It is shown in the results that under normal situations, routes found by the new strategy are approximately similar to that found by traditional DFS algorithms. However, the new strategy is more conservative when calculating the time cost of routes with discrete speed distributions and with extreme low or high real time speed values, thus guaranteeing the level of reliability. The strategy is presented in this paper with the level of reliability of 90 percent. It is essential to note that the level is not supposed to be fixed, and it can be determined based on real demands.

This paper has presented a concept of modified speed value in order to take into account the uncertainties that may be encountered during the emergency management procedure. The modification function is established based on several empirical concepts presented in 3.3, which indicates that improvements can be made by studying and simulating the regulations of speed changes with disparate lengths of time, and presenting a more adequate and precise modification function. Meanwhile, in the experimental implementation system, simplifications are made to acquire a fast calculation speed, but it is evident that speed information of the same types of roads cannot be exactly the same. Consequently, more complex situations should be considered, and speed information of each road should be distinguished and used separately to validate the strategy. Additionally, the route planning strategy in this paper didn't account for traffic flow on intersections that can actually exert substantial influence on the time cost calculated in the algorithms, and future work should investigate the feature of intersections and combine them with dynamic and uncertain factors as well.

\section{References}

1. G. Theodoulou and B. Wolshon, Modeling and analyses of freeway contra flow to improve future evacuations, in Proceedings of the 83rd Annual Meeting of the Transportation Research Board (CD-ROM) (Washington, D.C., United States, 2004).

2. P. M. Murray-Tuite and H. S. Mahmassani, Model of household trip chain sequencing in an emergency evacuation. Transportation Research Record: Journal of the Transportation Research Record. 1831(2003)21-29.

3. H. Q. Fu and C. Wilmot, A sequential logit dynamic travel demand model for hurricane evacuation, in Proceedings of the 83rd Annual Meeting of the Transportation Research Board (CD-ROM) (Washington, D.C., United States, 2004).

4. F. Southworth, Regional evacuation modeling: A state-of-the-art review (Oak Ridge National Laboratory, Oak Ridge, 1991).

5. E. Urbina and B. Wolshon, National review of hurricane evacuation plans and policies: A comparison and contrast of state practices. Transp. Res., Part A: Policy Pract. 37 (3)(2003)257-275

6. R. Alsnih and P. R. Stopher, A review of the procedures associated with devising emergency evacuation plans, in Proceedings of the 82nd Annual Meeting of the Transportation Research Board (CD-ROM) (Washington, D.C., United States, 2003).

7. H. Liu, J. Ban, W.T. Ma and P. Mirchandani, Model reference adaptive control framework for real-time traffic management under emergency evacuation, in Proceedings of the 85nd Annual Meeting of the Transportation Research Board (CD-ROM) (Washington, D.C., United States, 2007).

8. B. Barrett, B. Ran, and R. Pillai, Developing a dynamic traffic management modeling framework for hurricane evacuation. Transportation Research Record: Journal of the Transportation Research Record. 1773(2000) 115-121.

9. T. Cova and J. Johnson, A network flow model for lane-based evacuation routing. Transp. Res., Part A: Policy Pract. 37(7) 579-604.

10. X. Chen, Q. Fei and W. Li, A new shortest path algorithm based on heuristic strategy, in Proceedings of the 6th 
World Congress on Intelligent Control and Automation (Dalian, China,2006), pp:2531-2536.

11. I. Chabini and B. Dean, Shortest path problems in discrete-time dynamic networks: Complexity, algorithms, and implementations, Technical Report (Massachusetts Institute of Technology, 1999).

12. E. P. Hart, N. J. Nilsson and B. Raphael, A formal basis for the heuristic determination of minimum cost paths. IEEE Trans. Syst. Sci. Cybern. SSC-4(2) (1968)100-107.

13. V. Kumar, A. Grama, A. Gupta and G. Garypis, Introduction to Parallel Computing: Algorithm Design and Analysis (Benjamin Cummings/Addison Wesley, Redword City, 1994).

14. M. Dorigo, Optimization learning and natural algorithms, PhD thesis (Politecnico di Milano, 1992).

15. M. B. Chandra and R. Baskaran, Survey on Recent Research and Implementation of Ant Colony Optimization in Various Engineering Applications. International Journal of Computational Intelligence Systems. 4(4)(2011)566-582.

16. H. Q. Fu, and C. Wilmot, A sequential logit dynamic travel demand model for hurricane evacuation, in Proceedings of the 83rd Annual Meeting of the Transportation Research Board (CD-ROM) (Washington, D.C, United States, 2004).

17. S. Ugurlu and I. Kaya, A New Reliability Index Based on Fuzzy Process Capability Index for Travel Time in Multi-modal Networks. International Journal of Computational Intelligence Systems. 4(4)(2011)550-565.

18. R. P. Roess, E. S. Prassas and W. R. McShane, Traffic Engineering, 3rd edn. (Pearson College Div, Victoria, 2004).

19. V. F. Hurdle, M. I. Merlo and R. Doug, (1997), Study of speed flow relationships on individual freeway lanes. Transportation Research Record: Journal of the Transportation Research Record. 1591(1997)7-13.

20. Li, R. M, Research on Urban Emergency Management System, Technical Report (Tsinghua University Department of Civil Engineering, 2007). 